cess and 1.00:1.46 when the ferrocyanide was in excess. These results are in accord with those given on page 825 and are at variance with those of Stone and Van Ingen.

As the solution obtained by treating the zinc ferrocyanides with ammonia undergoes decomposition with the formation of blue precipitates and soluble ferricyanides, it is intended to continue the investigation of the action of ammonia on the potassium cadmium ferrocyanides instead of on the zinc salts.

QUANTITATIVE LABOKATORY,

June 4,1902 .

\title{
ON STANDARD TARTAR EMETIC AND ITS STRUCTURAL FORMULA.
}

\author{
BY F, E. HALE,
}

keceived May z3, I902.

GRUENER ${ }^{1}$ has already shown that tartar emetic solutions containing about 16 grams of tartar emetic, 20-30 grams of tartaric acid, and I-IO cc. of concentrated hydrochloric acid to the liter, will keep from five to twelve months without any change in strength. There is no deposit of antimonious oxide under these conditions, no oxidation, and no signs of fungous growth. Gruener determined the strength of his tartar emetic solutions by titration with a decinormal iodine solution, standardized by decinormal arsenite. The mean of twenty-nine determinations showed 43.95 per cent. of antimonious oxide in tartar emetic. Theory required 43.37 per cent. ( $\mathrm{Sb}=\mathrm{I} 2 \mathrm{O}, \mathrm{KSbOC}_{4} \mathrm{H}_{4} \mathrm{O}_{6} \cdot 1 / 2 \mathrm{H}_{2} \mathrm{O}=332$ ). The cause of this discrepancy between arsenite and tartar emetic solutions made up as standards according to the accepted molecvlar formulas is a matter of considerable interest. One suggested explanation of this difference has been found in the supposition that the end reaction between starch and iodine is delayed when titrating tartar emetic by iodine until an excess of iodine is present. I have already shown in a previous paper ${ }^{2}$ that a pure starch solution gives a sharp end reaction with both tartar emetic and arsenite solutions and that while with impure starch there is a loss of iodine accompanied by the production of reddish hues in titrating tartar emetic, as shown by the difference between the readings made in the presence of potassium iodide by the yellow color of 
iodine and by the blue of starch iodide, yet it is no greater than in titrating arsenite solution in the presence of an impure starch. If only an impure starch is available, the reading should be made without starch, for the presence of potassium iodide renders very sharp the yellow color of the first excess of free iodine. This first reading may be afterwards corroborated by adding the starch solution which will then give only a pure blue color. The above discrepancy must then be due to some other cause than delay of formation of the starch iodide. The present paper will prove that this discrepancy is due to the ease with which tartar emetic loses its water of crystallization and that in order to get a salt of the exact composition, $\mathrm{KSbOC}_{4} \mathrm{H}_{4} \mathrm{O}_{6} \cdot 1 / 2 \mathrm{H}_{2} \mathrm{O}$ (mol. wt. 332.15), certain conditions must be very closely observed.

In the previous paper ${ }^{1}$ a tartar emetic solution was used containing exactly I6 grams of tartar emetic to the liter, 25 grams of tartaric acid, and I cc. of concentrated hydrochloric acid. Of this solution $50 \mathrm{cc}$. were oxidized by $47.75 \mathrm{cc}$. of decinormal iodine solution. Now $49.48 \mathrm{cc}$. of the iodine solution oxidized $50 \mathrm{cc}$. of a decinormal arsenite solution. Therefore it should have taken $47.68 \mathrm{cc}$. of the iodine solution to oxidize $50 \mathrm{cc}$. of the tartar emetic solution; that is to say, the tartar emetic took 0.07 cc. of decinormal iodine solution more than the theory. Calculated on the ratio of antimony to tartar emetic used the error was $0.06+$ per cent. The tartar emetic was recrystallized from a boiling water solution, filtered, and air-dried. In the following experiments two different samples of commercial tartar emetic were used, which will be designated as $\mathrm{C}_{1}$ and $\mathrm{C}_{2}$. $\mathrm{C}_{1}$ was in crystalline condition, containing medium-sized crystals; $\mathrm{C}_{2}$ was in fine powdery form. Twelve different recrystallized products were used, designated as $R_{1}$, $R_{2}$, etc., to $R_{12}$. $R_{1}, R_{3}, R_{11}$ and $R_{12}$ were recrystallized from $C_{2}$, the others from $C_{1} ; R_{1}, R_{9}, R_{10}, R_{11}$ and $R_{12}$ were in mediumsized crystals from $1 / 32$ to $1 / 4$ of an inch in size. By rapid stirring, $R_{2}$ and $R_{3}$ were crystallized in very fine condition. $R_{4}, R_{5}$, $R_{8}, R_{7}$ and $R_{8}$ were crystallized in exceedingly fine condition by precipitation by alcohol with rapid stirring. The attempt was made to get both a definite crystalline salt and a definite anhydrous salt.

Decinormal solutions were made as follows: Exactly I6.6075

1 Loc. cit. 
grams of the crystalline tartar emetic (332.15) or I6.1 575 grams of the anhydrous salt (323.I5) were weighed out and dissolvedina little water by the aid of a filtered solution of 25 grams of tartaric acid. This solution, upon dilution, sometimes gave a crystalline precipitate which, however, completely redissolved upon further dilution and the addition of $5 \mathrm{cc}$. of concentrated hydrochloric acid. The solution was then diluted to the liter mark. No heat was necessary in making the solutions, a thorough shaking being sufficient to dissolve the tartar emetic in the tartaric acid. The strength of the solutions was determined by decinormal iodine solutions, containing 12.685 grams of iodine and 18 grams of potassium iodide to the liter. The iodine solutions were standardized by carefully prepared decinormal arsenite solutions, containing exactly 4.95 grams of resublimed arsenious oxide, dissolved by the aid of 3 grams of pure potassium hydroxide or of $70 \mathrm{cc}$. of a saturated solution of acid potassium carbonate. For titration a few cubic centimeters of the saturated solution of acid potassium carbonate were added.

From the recrystallized salt, $\mathrm{R}_{2}$, five different decinormal solutions were made, two in liter flasks and three in half-liter flasks.

The solutions in Table I, division $A$, were made from the freshly precipitated crystals; those in $B$ after the crystals, which were in fine condition, had stood several weeks. The experiments in $A$ show that the finely crystalline tartar emetic was dried just to the point at which, if made up at once into decinormal solutions, it agreed exactly with the decinormal arsenite solution. After some weeks' standing, though in a closed glass vessel, the preparation. as shown in $B$, had increased in the proportion of its antimony; that is, had lost water of crystallization.

Another recrystallization $\left(\mathrm{R}_{3}\right)$ was made from another sample of the commercial salt $\left(\mathrm{C}_{2}\right)$ and dried by exposure to the air. Decinormal solutions were made up at two different stages of the clrying, and demonstrate the ease with which tartar emetic loses its water of crystallization. The crystals were exposed to sunlight for a very short time and then laid in the shade. After an hour and three-quarters the first decinormal solution was made up and titrated. The drying in the air was continued for about four hours, and a half-liter decinormal solution was made up anci titrated. It is very obvious that after drying for an hour and 
three-quarters the tartar emetic was not completely dry, but that after six hours' drying the fine crystals had lost some of their water of crystallization.

TABLE I.

Final volume, I25 cc. $R_{2}$ from $C_{1}$.

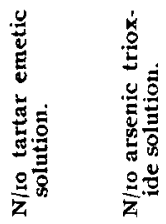

cc.

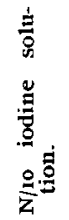

cc.

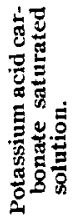

cc.

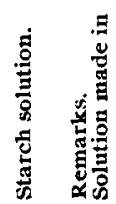

cc.

Color.

$A$. (Two solutions made from the freshly recrystallized tartar emetic.)

\begin{tabular}{|c|c|c|c|c|c|c|}
\hline I) & .. & 50 & 49.28 & 5 & I & medium blue (purplish) \\
\hline (2) & 50 & $\cdots$ & 49.28 & 25 & I) I-liter & deep blue \\
\hline 3) & 50 & . & 49.29 & 25 & I flask & medium blue (purplish) \\
\hline 4) & $\cdots$ & 50 & 49.32 & 5 & I & medium blue \\
\hline 5) & 50 & $\cdots$ & $49 \cdot \dot{3}^{2}$ & 25 & I) I/2-lite & good blue \\
\hline 6) & 50 & .. & 49.30 & 25 & I flask & good blue \\
\hline 7) & .. & 50 & $49 \cdot 31$ & 5 & I & medium blue (purplish) \\
\hline
\end{tabular}

$B$. (Three solutions made after the crystals had stood several weeks.)

\begin{tabular}{|c|c|c|c|c|c|c|}
\hline ( 8 ) & 50 & .. & 49.60 & 25 & I I-liter flask & good blue \\
\hline (9). & 50 & .. & 49.60 & 25 & I) I/2-liter & good blue \\
\hline (10) & 50 & $\cdots$ & $49 \cdot 5^{8}$ & 25 & I $\}$ flask & good blue \\
\hline (II) & $5^{\circ}$ & $\cdots$ & 49.60 & 25 & I ) I/2-liter & good blue \\
\hline (I2) & 50 & .. & 49.60 & 25 & I flask & good blue \\
\hline
\end{tabular}

TABIE II.

Final volume, $125 \mathrm{cc}$. $\mathrm{R}_{3}$ from $\mathrm{C}_{2}$.

\begin{tabular}{|c|c|c|}
\hline 总 & $\frac{1}{\bar{g}}$ & 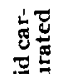 \\
\hline 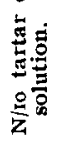 & 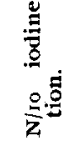 & ن \\
\hline cc. & cc. & cc. \\
\hline 50 & 49.23 & 25 \\
\hline 50 & 49.24 & 25 \\
\hline 50 & 49.46 & 25 \\
\hline
\end{tabular}

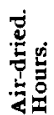

I $3 / 4 \quad$ I-liter flask

....

6

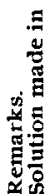

Color.

(13) $50 \quad 49.23$

(14) $50 \quad 49.24$

(I5) 50

For various reasons, the method of experimentation was changed to the following: Exactly 0.5 gram of tartar emetic was weighed out, dissolved in $50 \mathrm{cc}$. of water by the aid of $\mathrm{I}$ gram of tartaric acid and titrated by decinormal iodine, after the addition of $25 \mathrm{cc}$. of a saturated solution of acid potassium carbonate. The first experiments were made with two samples of recrystallized tartar emetic and with two samples of commercial tartar emetic. 
TABLE III.

Final volume, $120 \mathrm{cc}$.

(I6)
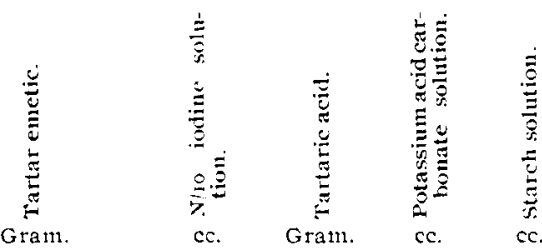

Color.

\begin{tabular}{|c|c|c|c|c|c|c|c|}
\hline (I6) & $\mathrm{R}_{1}$ & 0.5 & $\left\{\begin{array}{l}29.85 \\
29.85\end{array}\right.$ & I & 25 & 1 & deep blue \\
\hline ( 17$)$ & $\mathrm{R}_{1}$ & 0.5 & $\left\{\begin{array}{l}29.82 \\
29.85\end{array}\right.$ & I & 25 & I & $\begin{array}{l}\text { faint color } \\
\text { deep blue }\end{array}$ \\
\hline (18) & $\mathrm{R}_{1}$ & 0.5 & $\left\{\begin{array}{l}29.82 \\
29.8_{5}\end{array}\right.$ & I & 25 & I & $\begin{array}{l}\text { faint color } \\
\text { deep blue }\end{array}$ \\
\hline (Ig) & $R_{2}$ & 0.5 & $\left\{\begin{array}{l}29.82 \\
29.84\end{array}\right.$ & I & 25 & I & $\begin{array}{l}\text { faint color } \\
\text { deep blue }\end{array}$ \\
\hline (20) & $\mathrm{R}_{2}$ & 0.5 & 29.88 & I & 25 & I & very deep blue \\
\hline (2I) & $\mathbf{R}_{2}$ & 0.5 & 29.84 & 1 & 25 & $I$ & medium blue \\
\hline (22) & $\mathrm{C}_{1}$ & 0.5 & $30.3 \mathrm{~S}$ & I & 25 & I & medium blue \\
\hline (23) & $c_{1}$ & 0.5 & $\left\{\begin{array}{l}30.31 \\
30.34\end{array}\right.$ & I & 25 & I & $\begin{array}{l}\text { pale purple } \\
\text { deep blue }\end{array}$ \\
\hline (24) & $c_{1}$ & 0.5 & $3^{0.3^{8}}$ & I & 25 & I & deep blue \\
\hline (25) & $\mathrm{C}_{2}$ & 0.5 & $30.3^{8}$ & I & 25 & I & deep blue \\
\hline (26) & $\mathrm{C}_{2}$ & 0.5 & $\left\{\begin{array}{l}30.5^{\circ} \\
30.5^{2}\end{array}\right.$ & I & 25 & I & $\begin{array}{l}\text { pale purple } \\
\text { deep blue }\end{array}$ \\
\hline$(27)$ & $\mathrm{C}_{2}$ & 0.5 & 30.44 & I & 25 & I & deep blue \\
\hline
\end{tabular}

$\mathrm{R}_{1}$, of medium-sized crystals, though kept for several months, agreed with the freshly recrystallized tartar emetic $R_{2}$. The two commercial samples appeared to have lost water of crystallization, or contained impurity, and $\mathrm{C}_{2}$, which was in fine powdery condition, appeared to have lost more water than $\mathrm{C}_{1}$, which was in medium-sized crystals. A difference in the method of commercial preparation may have been responsible for this discrepancy. That recrystallization was necessary in order to make up a standard solution was evident.

The effect of desiccators was next tried upon the recrystallized tartar emetic. A sample of $R_{1}$, finely pulverized, was placed in a desiccator containing sulphuric acid and another sample of the same preparation was put in a vacuum desiccator over sulphuric acid. The action of the two clesiccators seemed to be comparable in extent and rapidity, and by comparison with experiments (I6), ( 17 ), and (I8) it will be seen that there is a gradual and slow loss of water of crystallization. 
TABLE IV.

Final volume, I20 cc. $R_{1}$.

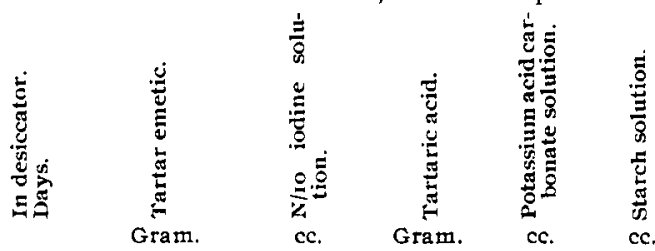

Color.

(In sulphuric acid desiccator.)

$\begin{array}{rrrrrrrr}(28) & 4 & 0.5 & 29.98 & \text { I } & 25 & \text { I } & \text { deep blue } \\ 29 & 7 & 0.5 & 30.0 I & \text { I } & 25 & \text { I } & \text { deep blue } \\ (30) & \text { I } & 0.5 & 30.16 & \text { I } & 25 & \text { I } & \text { medium blue } \\ 3 \mathrm{I} & 60 & 0.5 & 30.38 & \text { I } & 25 & \text { I } & \text { pale blue }\end{array}$

$\begin{array}{llllllll}(32) & 4 & 0.5 & 29.98 & \text { I } & 25 & \text { I } & \text { deep blue } \\ \text { (33) } & 7 & 0.5 & 29.94 & \text { I } & 25 & \text { I } & \text { deep blue }\end{array}$ $\mathbf{N} /$ to arsenic trioxide solution.

(34) $\quad$.

50

$49.3 \mathrm{I}$

$5 \quad \mathrm{I}$

deep blue

The hot air-bath was then resorted to with the most varied results. Portions of $R_{1}$ and of $C_{1}$ and $C_{2}$ were heated in the airbath. The two latter samples were taken to see whether the commercial salt could be reduced to anhydrous condition completely and agree with the recrystallized salt. The sample of $R_{1}$, in experiment ( 35 ), was heated at $120^{\circ}$ for an hour and between $120^{\circ}$ and $95^{\circ}$ for three hours. The same sample was then reheated with samples of the commercial preparations and with a fresh sample of $R_{1}$ at $104^{\circ}-110^{\circ}$ for two and one-half hours, I I $6^{\circ}-130^{\circ}$ for two hours, and I I $5^{\circ}$-I IO ${ }^{\circ}$ for three hours.

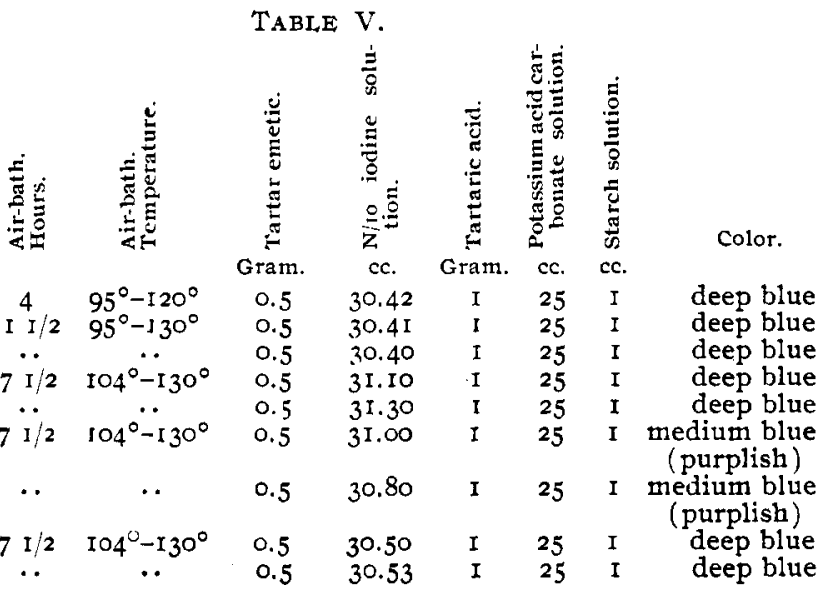


The reheated sample, (36) and (37), was not changed by the second heating, but lost considerable water by the first heating. It stood in a desiccator between the two periods of heating. The fresh sample of the same preparation, (38) and (39), lost most heavily of all, and was closely followed by the more crystalline commercial sample $\mathrm{C}_{1},(40)$ and $(4 \mathrm{I})$, which had been powdered before heating. The finely powdered commercial sample $\mathrm{C}_{2},(42)$ and (43), lost but little water.

The sample $C_{1}$ which had now been heated already seven and one-half hours from $104^{\circ}$ to $130^{\circ}$ was reheated for three hours at $I 60^{\circ}-I 65^{\circ}$, and a fresh sample of $R_{1}$ was heated at $160^{\circ}-165^{\circ}$ for two hours. Again the fresh sample lost more water than the sample previously heated.

TABLE VI.

Final volume, I $20 \mathrm{cc}$.

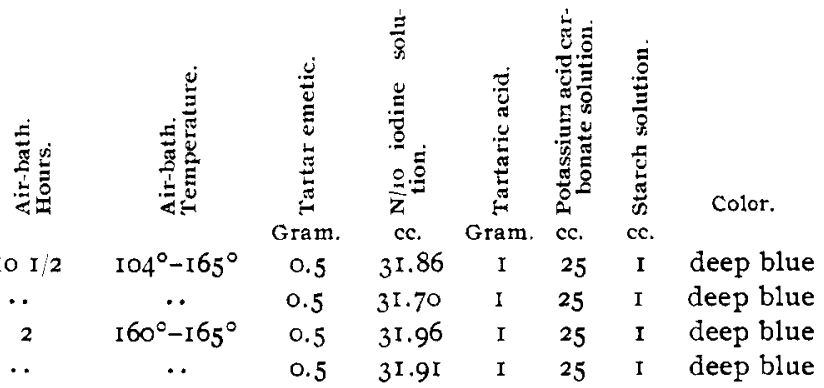

A still greater loss of water is evident in these experiments, and in this table, as well as in experiments (38) to (4I) inclusive, in Table $V$, the discrepancy between the duplicates was due to the difficulty of weighing the portions exactly, since the dried tartar emetic took up moisture so rapidly on the balance.

In Table VII the molecular weights of tartar emetic are given, calculated from the amounts of antimony present as shown by the iodometric titration of the preceding experiments. The decinormal arsenite solution was taken as standard.

A study of the preceding tables in the light thrown upon them by Table VII leads to some interesting results. The molecular weights run in a series from that of crystalline tartar emetic down almost to the theoretical state in which the crystalline salt has lost I.5 molecules of water, passing through all intermediate stages, but never surely resting at any one spot. Two important stages 
TABLE VII.

$49.29 \mathrm{cc}$. (absolute amount) of $\mathrm{N} / \mathrm{ro}$ iodine solution $=50 \mathrm{cc}$. of $\mathrm{N} / \mathrm{10}$ arsenic trinxide solution.

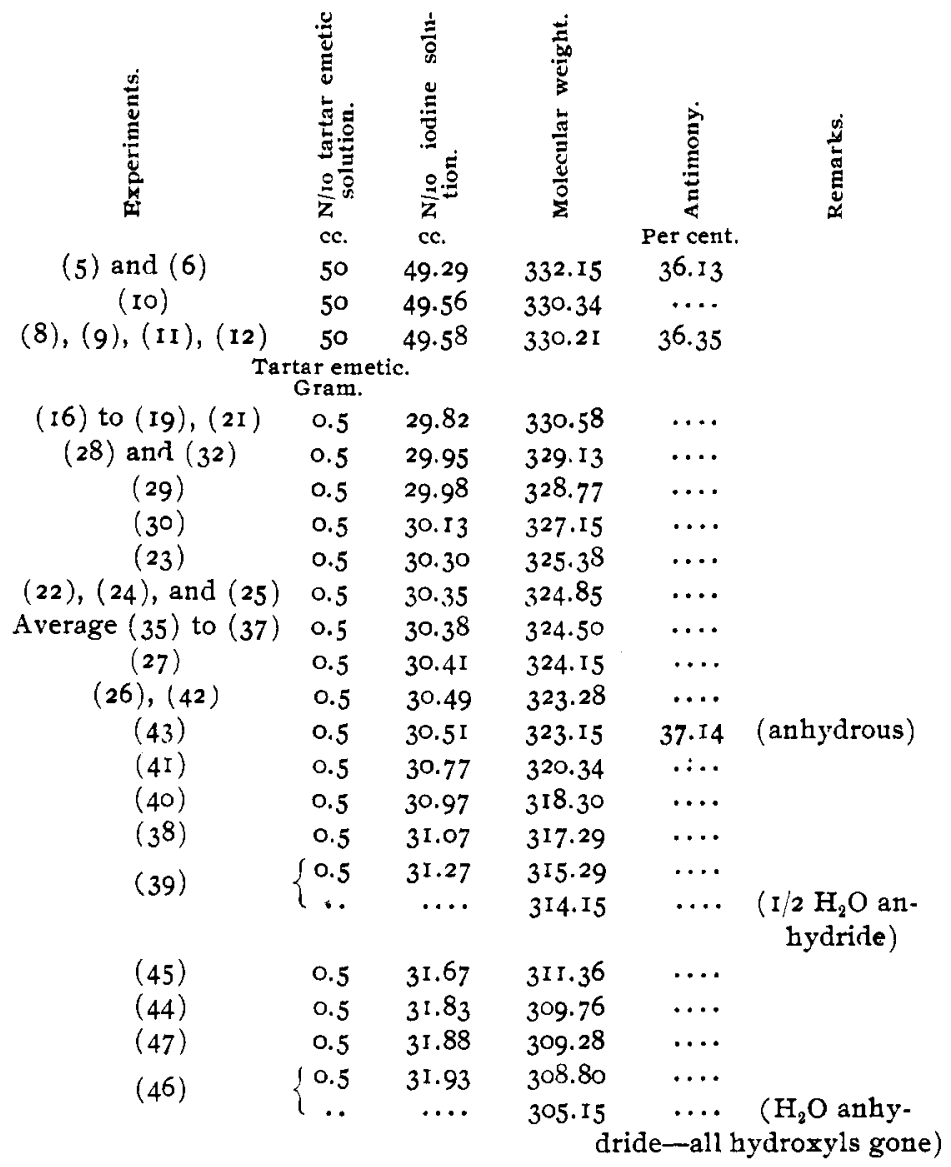

are reached: when all the water of crystallization is gone, the anhydrous state, and when 0.5 molecule of water further has been lost, the first anhydride stage. The greatest error met with in the recrystallized tartar emetic, if air-dried, is about $0.2+$ per cent., calculated on the ratio of antimony to tartar emetic, and that after standing in fine condition in a closed bottle for several weeks. After standing for about two weeks in a sulphuric acid desiccator the finely recrystallized tartar emetic lost almost exactly one-half of its water of crystallization, and after two months almost all of 
its water of crystallization. The commercial salt $\left(\mathrm{C}_{2}\right)$ had but little water of crystallization and lost it all upon heating in the air-bath, but went no further even at the temperature of $130^{\circ}$. Though heated at $95^{\circ}-120^{\circ}$ for four hours, the recrystallized salt $\left(R_{1}\right)$ failed to lose all of its water of crystallization, and reheating for seven and a half hours at $104^{\circ}-130^{\circ}$ failed to produce any further loss. Especially interesting are the results of heating the fresh samples at the higher temperatures. Between $120^{\circ}$ and $130^{\circ}$ $\mathrm{R}_{1}$ and $\mathrm{C}_{1}$ (in crystalline form) lost water beyond the anhydrous state and approached the anhydride $\left(\mathrm{KSbOC}_{4} \mathrm{H}_{4} \mathrm{O}_{6}-1 / 2 \mathrm{H}_{2} \mathrm{O}\right)$. Upon heating at the temperature $160^{\circ}-165^{\circ}$, the same preparations lost yet more water and approached the state of a double anhydride in which all the hydroxyls were gone $\left(\mathrm{KSbOC}_{4} \mathrm{H}_{4} \mathrm{O}_{6}-\mathrm{H}_{2} \mathrm{O}\right)$. The anhydride state was also indicated by the readiness of the salts, after such heating, to take up water again while on the balance. Both of these anhydrides have been previously obtained.

Liebig $^{1}$ states that, according to Dulk, Wallquist, and Brandes, and in accordance with his own experiments, tartar emetic loses its water of crystallization at $100^{\circ}$. By careful heating to $300^{\circ}$ he caused tartar emetic to lose, without charring, I molecule of water. Dumas and Piria ${ }^{2}$ established the fact that tartar emetic contains 0.5 molecule of water of crystallization to the accepted empirical formula. Their analyses were very close. They also heated tartar emetic to a high temperature. In an oil-bath it became brownish at $235^{\circ}-240^{\circ}$; at $220^{\circ}$ it was still white. They gave the following double formula: $\left(\mathrm{C}_{4} \mathrm{H}_{4} \mathrm{O}_{6}\right)_{2} \cdot(\mathrm{SbO})_{2} \cdot \mathrm{K}_{2} \cdot \mathrm{H}_{2} \mathrm{O}$; at $100^{\circ}\left(\mathrm{C}_{4} \mathrm{H}_{4} \mathrm{O}_{6}\right)_{2} \cdot(\mathrm{SbO})_{2} \cdot \mathrm{K}_{2}$; at $220^{\circ}\left(\mathrm{C}_{4} \mathrm{H}_{2} \mathrm{O}_{5}\right)_{2} \cdot(\mathrm{SbO})_{2} . \mathrm{K}_{2}$. Ber$1 n^{3}$ states that at $100^{\circ}$ in a stream of dry air tartar emetic loses two-thirds of its water of crystallization very easily, but the rest with difficulty; that at a temperature slightly over $100^{\circ}$ the body changes, losing 0.5 molecule of water at $160^{\circ}-180^{\circ}$ or by longer heating at $\mathrm{I} 30^{\circ}$; that another half molecule of water goes at $200^{\circ}$ $220^{\circ}$, making the formula now $\mathrm{C}_{4} \mathrm{H}_{2} \mathrm{O}_{3}$. SbO.K.

A series of experiments was now made to reach the desired end by alcoholic precipitation. A concentrated, yet not saturated, solution of tartar emetic was made in boiling water and filtered into about an equal volume of 95 per cent. alcohol. The tartar

1 Ann. Chem. (Liebig), 26, 132.

2 Ibid., 44, 85.

3 Ibid., 64, 359 . 
emetic was precipitated as soon as it reached the alcohol and was frequently stirred. It was then filtered by suction, kept under suction for from five to ten minutes, and air-dried. The product was a very fine white fluffy powder. Four such recrystallizations were made at first $\left(R_{4}, R_{5}, R_{6}\right.$, and $\left.R_{7}\right)$ and dried for different lengths of time in the air. Half-gram portions were weighed out, dissolved in I gram of tartaric acid, and titrated by decinormal iodine, as in the previous experiments. The results are given in Table VIII.

$$
\begin{aligned}
& \text { TABLE VIII. }
\end{aligned}
$$

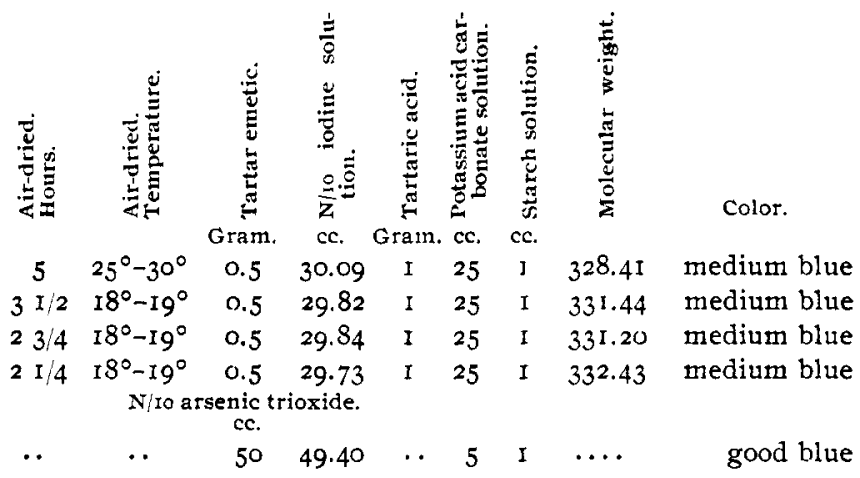

The iodine solution had stood unused for about a month and had grown weaker; hence the new standard was used in determining the molecular weights. The tartar emetic precipitated by alcohol, though containing water of crystallization, was in such fine condition that it lost some of its water of crystallization readily in the air, or else had been precipitated by the alcohol with an incomplete amount of water of crystallization. The infuence of time upon the results of air-drying is prominent. $R_{5}, R_{6}$, and $R_{7}$ came, however, so near to the theory that another preparation $\left(R_{8}\right)$ was made very carefully as follows:

A fairly concentrated solution of tartar emetic in $250-300 \mathrm{cc}$. of boiling water was filtered into $400 \mathrm{cc}$. of 95 per cent. alcohol, and the precipitate was filtered off by suction, washed twice by 95 per cent. alcohol, kept under good suction for fifteen minutes longer, and air-dried two and a half hours at $19^{\circ}-20^{\circ}$. A half liter of decinormal tartar emetic solution was made up at once and titrated. 
$(53)$

$(54)$

(56)
TABLE IX.

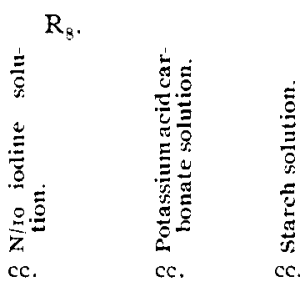

Color.

medium blue medium blue deep blue

One-half gram of the tartar emetic, dissolved in tartaric acid as above and titrated, took $29.80 \mathrm{cc}$. of decinormal iodine solu. tion.

The agreement between the arsenic and tartar emetic standards was fairly good, but hardly close enough to warrant the use of this mode of preparing tartar emetic for standardizing purposes. As the tartar emetic in this exceedingly fine form gave uniform results and was so ready to lose its water of crystallization, it was tried in the air-bath under closely regulated conditions.

TABLE X.

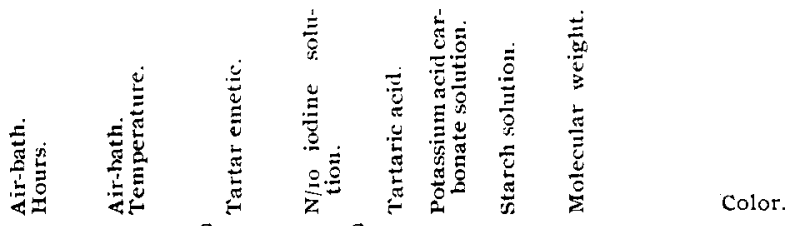

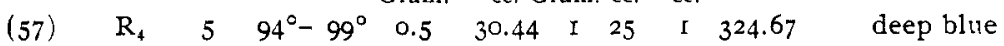

(58) $\quad \begin{array}{lllllllll}R_{5} & 5 & 94^{\circ}-99^{\circ} & 0.5 & 30.4 \mathrm{I} & \text { I } & 25 & \text { I } & \ldots\end{array}$

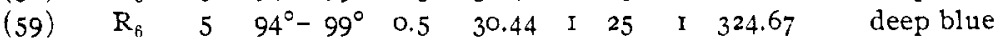

(60) $\begin{array}{lllllllll}R_{7} & 5 & 94^{\circ}-99^{\circ} & 0.5 & 30.48 & \text { I } & 25 & \text { I } & \ldots\end{array}$ deep blue

(6I) $\quad R_{+} \quad 3 \quad 96^{\circ}-99^{\circ} \quad 0.5 \quad 30.44$ I 25 I 324.67 deep blue

$\begin{array}{llllllllllll}(62) & \ldots & \ldots & \ldots & 50 & 49.42 & \ldots & 5 & \text { I } & \ldots & & \end{array}$ Tartar emetic.

(63) $\quad R_{4} \quad 7 \quad 97^{\circ}-103^{\circ} \quad 0.5 \quad 30.49$ I $25 \quad$ I $\quad \ldots . \quad$ deep blue

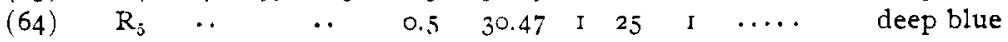

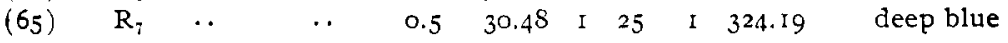

(66) $\quad \mathrm{R}_{4}, \mathrm{R}_{5}, \mathrm{R}_{6}, \mathrm{R}_{7}$

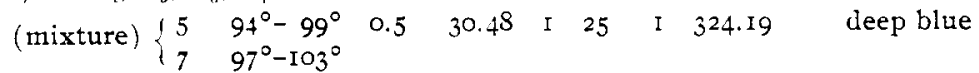

(67) $\mathbf{R}_{6}{ }^{*} \quad 3 \quad 97^{\circ}-103^{\circ} \quad 0.5 \quad 30.48$ I $25 \quad$ r 324.19 deep blue

* Stood in absolute alcohol for twenty-four hours, then was filtered and air-dried. 


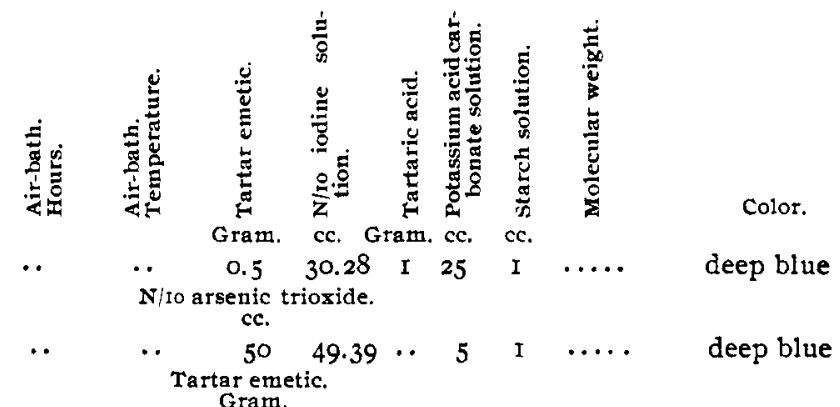

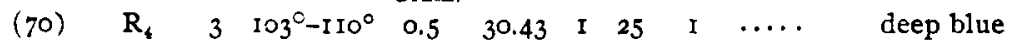

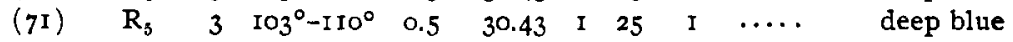

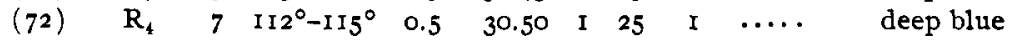

(73) $\begin{array}{lllllllll}R_{5} & 7 & \text { II } 2^{\circ}-115^{\circ} & 0.5 & 30.46 & \text { I } & 25 & \text { I } & \ldots .\end{array} \quad$ deep blue

(74) $\quad R_{4} \quad 5.5 \quad I I 4^{\circ}-I 2 I^{\circ} \quad 0.5 \quad .30 .48$ I 25 I $324.19 \quad$ deep blue

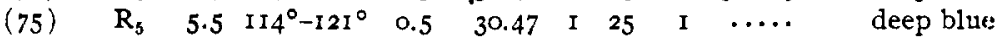

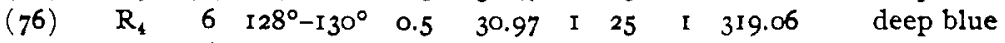

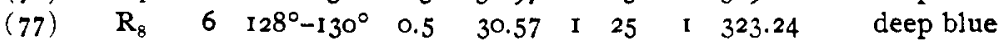

(78) $\quad \mathrm{R}_{4}, \mathrm{R}_{5}, \mathrm{R}_{6}, \mathrm{R}_{7}$

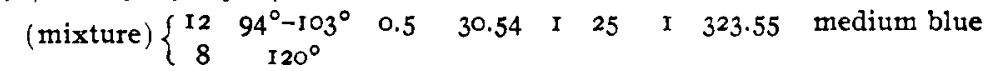

$\begin{array}{lllllllllll}\text { (79) } & R_{4} & 8 & I_{20} & 0.5 & 30.47 & \text { I } & 25 & \text { I } & \ldots & \text { medium blue }\end{array}$ $\mathrm{N} /$ io arsenic trioxide.

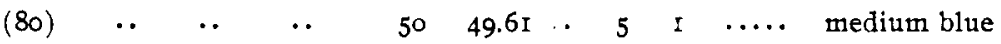
Tartar emetic.

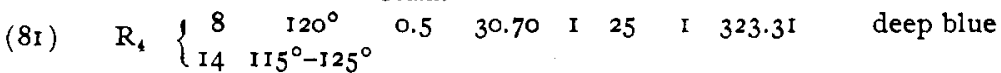

(82) $\quad R_{8}\left\{\begin{array}{ccccccccc}8 & I_{20^{\circ}} & 0.5 & 30.57 & \text { I } & 25 & I & \cdots & \text { Ieep biue } \\ 14 & I_{15} 5^{\circ}-125^{\circ}\end{array}\right.$

(83) $\quad R_{4}, R_{5}, R_{6}, R_{7}$

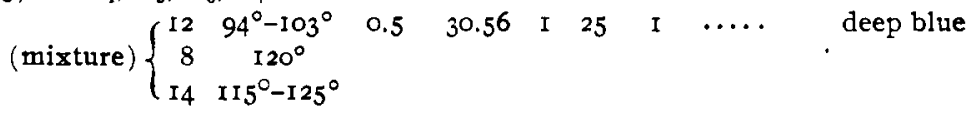

From three to five hours' drying at $94^{\circ}-99^{\circ}$ failed to dry the tartar emetic completely to the anhydrous state (323.I5). The experiments $(63)$ to $(75)$, made at temperatures varying about $5^{\circ}$ from $100^{\circ}$ to $125^{\circ}$, carried the drying slightly further, but still the molecular weight of tartar emetic was a unit too high for the anhydrous condition. Experiment (68) shows the effect of absolute alcohol upon the water of crystallization in twenty-four hours. In $(67)$ this same preparation lost in three hours in the air-bath about the same amount of water as the others in a longer time.

* Stood in absolute alcohol for twenty-four hours, then was filtered and air-dried. 
In (66) the portions heated in (57) to $(6 \mathrm{I})$ were reheated, making twelve hours of total heating. This same mixture was again reheated in (78) and (83). In (73) the tartar emetic became almost entirely anhydrous according to theory, but this temperature, $128^{\circ}$ I $30^{\circ}$, is on the danger line, since in the duplicate experiment, $(76)$, the tartar emetic passed slightly beyond the anhydrous condition to that of an anhydride. Experiments $(78)$ to $(83)$ show the effect of long heating at I $15^{\circ}-125^{\circ}$. In $(78)$ to $(8 \mathrm{I})$ the portions were almost anhydrous, but the other samples fell short. The difference between the readings in experiments $(8 \mathrm{I})$ to $\left(8_{3}\right)$ and those of the preceding experiments was due to the use of a new decinormal iodine solution. Since in these last experiments twenty-two to thirty-four hours' drying at I I $5^{\circ}-125^{\circ}$ failed to bring uniform and sufficiently exact results, it is hardly possible to make up standard decinormal tartar emetic solutions from the product obtained by drying in the air-bath, and the method of precipitation by alcohol yields the most susceptible form of tartar emetic for this purpose. This conclusion is exemplified in Table XI. A decinormal solution of tartar emetic was made up in a $500 \mathrm{cc}$. flask from a mixture of the products in experiments $(57)$ to $(6 \mathrm{I})$ and another from the products dried at $100^{\circ}-\mathrm{I} 20^{\circ}$. These solutions were made on a basis of 323.15 (molecular weight of anhydrous tartar emetic) $i$. c.. I6. 575 grams per liter.

\section{TABLE XI.}

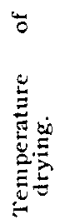

(84)

(85)
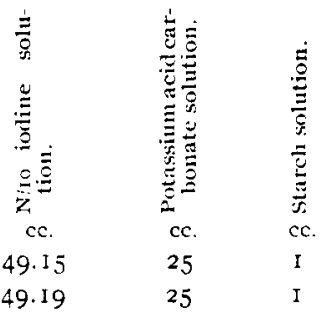

Color.

medium blue nedium blue

If in experiment (84) the value of 49.15 be taken as standard, then the molecular weight for $30.4 \mathrm{I}$, absolute decinormal iodine value obtained for 0.5 gram of this preparation in experiments (57) to $(6 \mathrm{I})$, is 332.08 . Therefore the decinormal solution agreed with the half-gram values, but both were too low as the decinormal arsenite standard of the iodine solution was 49.40 . The portion dried at $100^{\circ}-120^{\circ}$ likewise fell short of the arsenite 
standard, which was made certain by two separately prepared decinormal arsenite solutions.

The effect of drying in a sulphuric acid desiccator was also tried upon the product precipitated by alcohol. The last traces of water of crystallization appear to be exceedingly difficult to drive out.

TABLE XII.

(86) $\begin{array}{cc} & \\ & \\ \text { Gram. } & \text { cc. } \\ \text { I } & 25 \\ \text { I } & 25 \\ \text { I } & 25 \\ \text { I } & 25 \\ \text { I } & 25 \\ \text { I } & 25\end{array}$

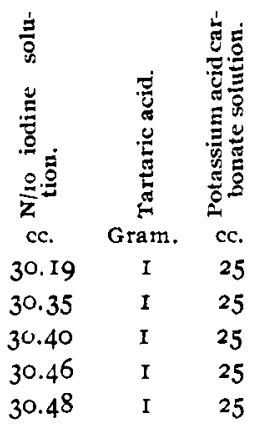

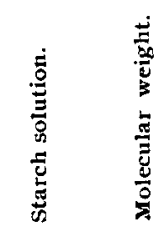

$\frac{2}{3}$

Color.

medium blue deep blue deep blue medium blue medium blue

As a last resource, the tartar emetic was recrystallized in medium-sized crystals, and air-dried. It will be remembered that the first recrystallization was made in this manner and that medium-sized crystals showed far less tendency to lose water of crystallization than the finely crystallized product. Four preparations were made up, two apiece from two commercial samples, and upon two different days. In about $300 \mathrm{cc}$. of boiling water enough tartar emetic was dissolved to make a concentrated, but not saturated, solution, which was filtered into flat crystallizing dishes and allowed to crystallize over night. The crystallization should not be too rapid. The crystals were filtered off by suction, washed twice with distilled water, and kept under suction for about five to ten minutes additional. They were then airdried from one and a half to four hours at a room temperature of $19^{\circ}-24^{\circ}$. The air was clear and dry. The crystals were pulverized and decinormal solutions made up in the usual manner in a half-liter flask.

These results, as close as could reasonably be expected, are given just as they came. No inferior experiments have been omitted. It is possible, by working with care, to make up decinormal tartar emetic solutions agreeing with decinormal arsenite by recrystallizing the tartar emetic slowly in medium-sized crystals, $1 / 32$ to $1 / 4$ of an inch in size, washing well with distilled water, 


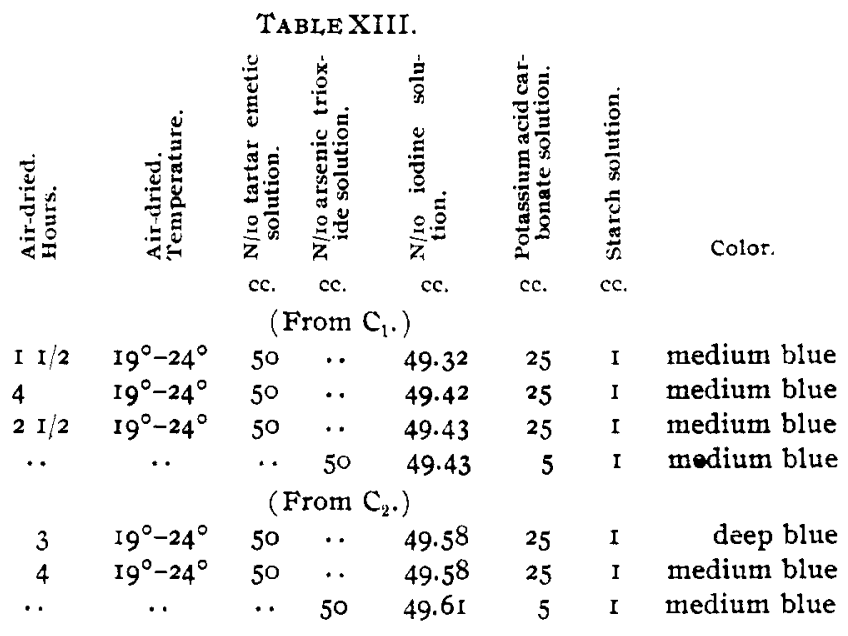

\begin{tabular}{|c|c|c|c|c|c|c|c|c|c|}
\hline (9I) & $\mathrm{R}_{3}$ & I $1 / 2$ & $19^{\circ}-24^{\circ}$ & 50 & $\cdots$ & $49 \cdot 3^{2}$ & 25 & I & medium blue \\
\hline (92) & $\mathrm{R}_{9}$ & 4 & $19^{\circ}-24^{\circ}$ & 50 & .. & 49.42 & 25 & I & medium blue \\
\hline (93) & $\mathrm{R}_{10}$ & $21 / 2$ & $19^{\circ}-24^{\circ}$ & 50 & $\cdots$ & 49.43 & 25 & I & medium blue \\
\hline (94) & $\cdots$ & .. & .. & . & 50 & 49.43 & 5 & I & modium blue \\
\hline \multicolumn{10}{|c|}{ (From $\mathrm{C}_{2}$. ) } \\
\hline (95) & $\mathrm{R}_{11}$ & 3 & $19^{\circ}-24^{\circ}$ & 50 & . & 49.58 & 25 & I & deep blue \\
\hline (96) & $R_{12}$ & 4 & $19^{\circ}-24^{\circ}$ & 50 & .. & $49.5^{8}$ & 25 & I & edium blue \\
\hline (97) & .. & .. & .. & .. & 50 & $49.6 I$ & 5 & I & medium blue \\
\hline
\end{tabular}

removing most of the moisture by about ten minutes' good suction, and air-drying from two and a half to four hours. The crystals should not lie exposed to the air for many hours after they are dry, and for exact work should be freshly prepared. On a damp day it might take longer to dry them than the time given above, but heat should never be used. The laboratory temperature should not range above $25^{\circ}$ and should be preferably lower. The crystals in the above preparations were considered dry when they showed not the slightest tendency to cling to a glass rod and were left exposed for an hour or two longer to make certain.

It has already been suggested that the antimony in tartar emetic is not attached to the carboxyl end of tartaric acid but to the alcoholic end. Clarke and stallo, ${ }^{1}$ in 1880 , opposed the idea that the group $(-\mathrm{Sb}=\mathrm{O})$ existed in tartar emetic and suggested that tartar emetic was a salt of $\mathrm{SbO}_{3} \mathrm{H}_{3}$. They gave the formula $\mathrm{Sb}\left\{\begin{array}{l}\mathrm{C}_{4} \mathrm{H}_{4} \mathrm{O}_{6} \\ \mathrm{OK}\end{array}\right.$ for tartaric emetic. Clarke and Evans ${ }^{2}$ found it difficult to obtain distinct products of tartaric acid and antimonious acid, and suggested the possible existence of a row of salts.

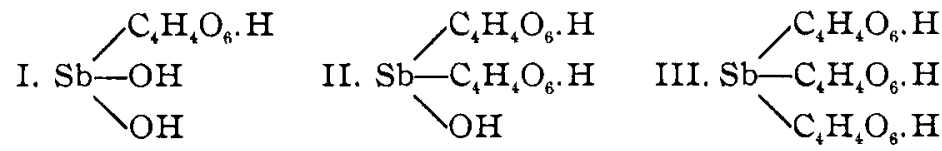

1 Ber. d. chem. Ges., 13, I788-1796.

2 loid, , 16, 2379-2387. 

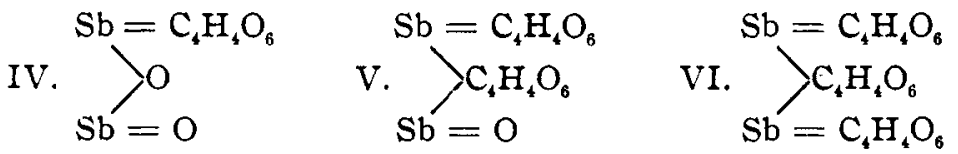

They thought they obtained in definite condition III, V, and VI, and apparently II. They changed the earlier published formula of tartar emetic to

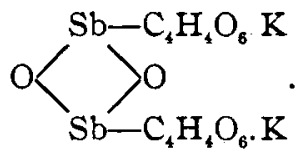

However, they make no distinct remark as to whether antimony is joined to the carboxyl or hydroxyl end of tartaric acid, but the idea of the acid function of antimony suggests the latter linking. Duve, ${ }^{1}$ in I869, claimed that boric acid united at the hydroxyl end of salts of tartaric acid. He believed that he separated the three following bodies:
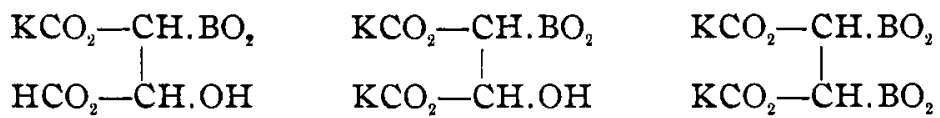

Adam $^{2}$ gives several reasons for believing that antimony plays the part of an acid and not of a base in tartar emetic. Of these the following seem to be of the greatest weight.

First. Bodies analogous to antimonious oxide react with alcoholic hydroxyls of organic bodies, e. g., $\mathrm{C}_{4} \mathrm{H}_{2} \mathrm{O}_{6} \mathrm{Na}_{2}(\mathrm{BoO})_{2}$ noted by Duve, and sodium borosalicylate described by himself. Sodium lactate reacts with boric acid, with antimonious oxide, and with arsenious oxide; potassium bimalate with boric acid, and with antimonious oxide; and Rochelle salt with one or two molecules of boric acid.

Second. Only those acids which possess a phenol or alcoholic function combine with antimonious oxide, boric acid, etc. $\mathrm{He}$, himself, established the fact that oxalic acid, phthalic acid, and their salts do not combine with boric acid.

Third. As a rule the free organic acid does not react with these bodies. Tertaric acid and antimonious oxide are an exception to this rule, however, as they do form unstable bodies. Klein ${ }^{3}$ showed that boric acid combines with salts of mucic acid and of

\footnotetext{
1 Jsb. d. Chem. (1869), p. 540.

2 Compi. rend., 118,1273 .

8 Compt. rend., 96, 1802 and 97, 1437.
} 
saccharic acid, but not with the free acid. He himselt noted inaction between salicylic acid and boric acid.

Fourth. Only organic salts or acids with a free hydroxyl group prevent the precipitation of ferric hydroxide by potassium hydroxide, e. g., lactic acid, malic acid, tartaric acid, salicylic acid, but not sodium borosalicylate. Monoborotartrate also prevents precipitation.

Fifth. Upon adding alkali, acid potassium carbonate, to tartar emetic heat is given off at once, but antimonious oxide is but gradually and slowly precipitated.

Henderson and Ewing, ${ }^{1}$ in 1895 , prepared various salts of arsenious oxide and tartaric acid and studied the action of arsenious oxide and antimonious oxide on citric, malic, lactic, and mucic acids. They also came to the conclusion that the antimonyl group $(-\mathrm{Sb}=\mathrm{O})$ displaces a hydroxyl group, but thought that the bodies formed were derivatives of antimonious acid as Clarke and Stallo ${ }^{2}$ suggested. They found that compounds of arsenious oxicle or of antimonious oxicle with citric acid, or with malic acid, which contain one hydroxyl group were composed of 1 atom of arsenic or of antimony to 2 molecules of the acid, that tartaric acid and mucic acid, having two and four hydroxyls respectively, combined in the ratio of I molecule of acid to I atom of antimony or of arsenic. They suggested the following formulas:

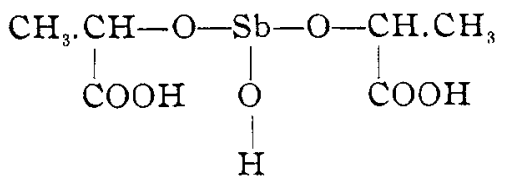

Antimoniolactic acid.

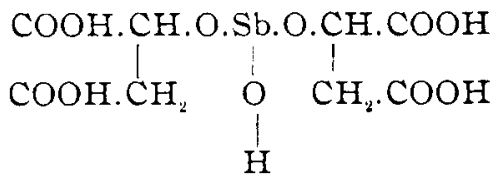

Antimoniomalic acid.<smiles>CCC(CC(=O)O)(O[Sb](O)OC(CC(=O)O)(CC(=O)O)C(=O)O)C(=O)O</smiles>

Antimoniocitric acid.

1 J. Chem. Soc. (London), 67, 102-109 and 10.30-1040.

: Loc. cit. 


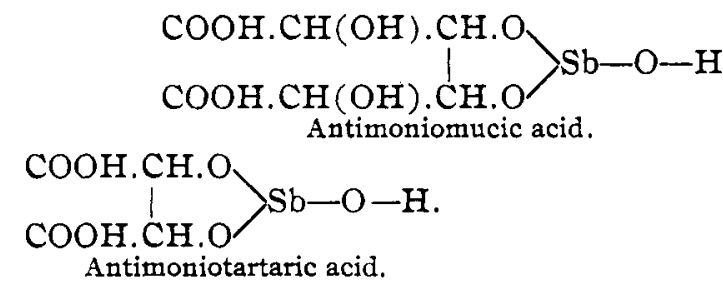

Their last formula, applied to tartar emetic, would not, however, explain the union of that water in the compound which is ordinarily called the water of crystallization. I would suggest for crystalline tartar emetic the double formula:

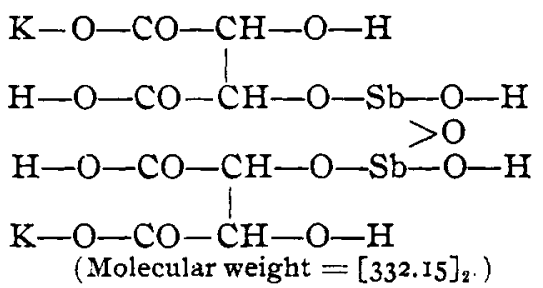

Tartaric acid forms an anhydride at $150^{\circ}$. The low temperature at which tartar emetic in the preceding experiments, and in accordance with the authors cited, lost 0.5 molecule of water between $130^{\circ}$ and $160^{\circ}$ would appear to be better explained by the formation of a tartar emetic anhydride than by a dehydration of the alcoholic groups according to the classic formula, or by such an action as Schiff, ${ }^{1}$ represents in the formula COOK. $\mathrm{C}_{2} \mathrm{H}_{2} \mathrm{O}_{2}=\mathrm{Sb}$.

Schiff ${ }^{2}$ calls tartaric acid tetrabasic.

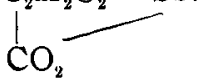

The various steps of dehydration of tartar emetic from the crystalline state, according to the formula proposed, might be represented as follows:

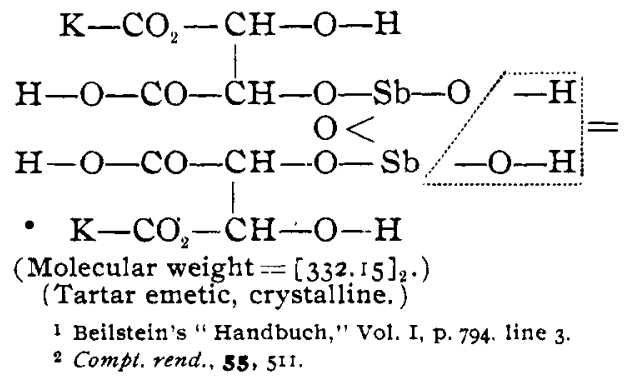


F. E. HALE.

$$
\begin{gathered}
\mathrm{K}-\mathrm{CO}_{2}-\mathrm{CH}-\mathrm{O}-\mathrm{H} \\
\mathrm{H}-\circlearrowleft-\mathrm{CO}-\mathrm{CH}-\mathrm{O}-\mathrm{Sb} \\
\mathrm{H}-\mathrm{O}-\mathrm{O}-\mathrm{CO}-\mathrm{CH}-\mathrm{O}-\mathrm{Sb}+\mathrm{H}_{2} \mathrm{O}= \\
\mathrm{K}-\mathrm{CO}_{2}-\mathrm{CH}-\mathrm{O}-\mathrm{H} \\
\left(\text { Molecular weight }=[323.15]_{2} .\right) \\
\text { (Tartar emetic, anhydrous.) }
\end{gathered}
$$

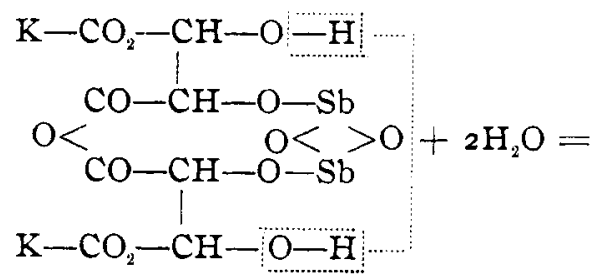

(Molecular weight $=\left[3 \mathrm{I}_{4} . \mathrm{I}_{5}\right]_{2}$.)

(Tartar emetic, anhydride.)

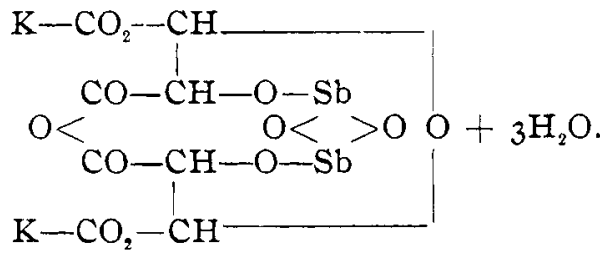

$$
\begin{aligned}
& \text { (Molecular weight } \left.=[305.15]_{2} .\right) \\
& \text { (Tartar emetic, double anhydride.) }
\end{aligned}
$$

The last reaction may be even better represented by doubling the equation and forming an anhydride between four molecules thus:

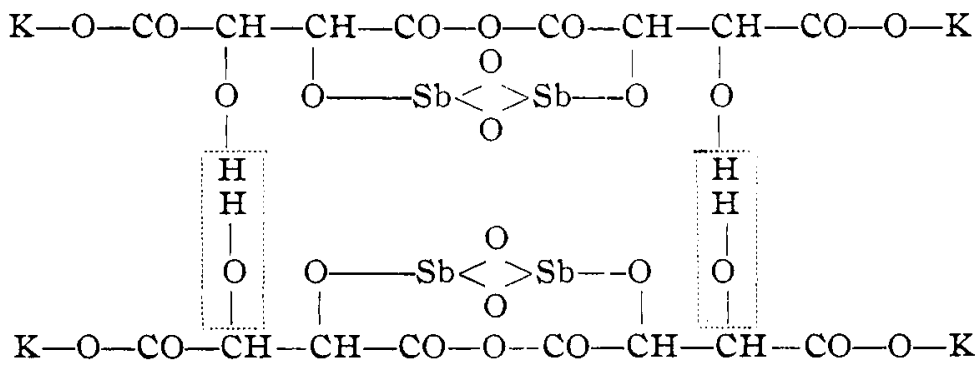

It is also possible that a lactone may form from the anhydrous salt instead of the further dehydration in steps thus: 


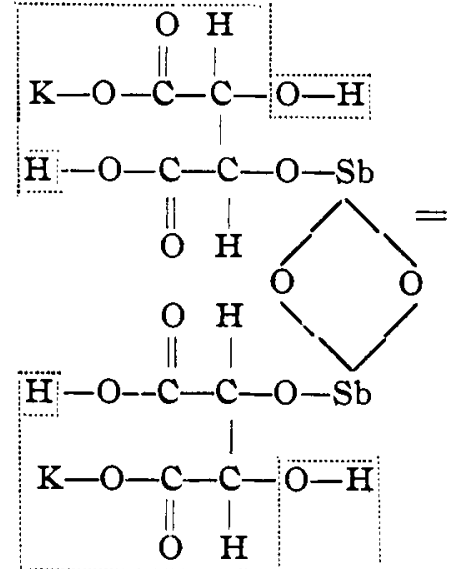

(Molecular weight $[323.15]_{2}$.) (Tartar emetic, anhydrous.)

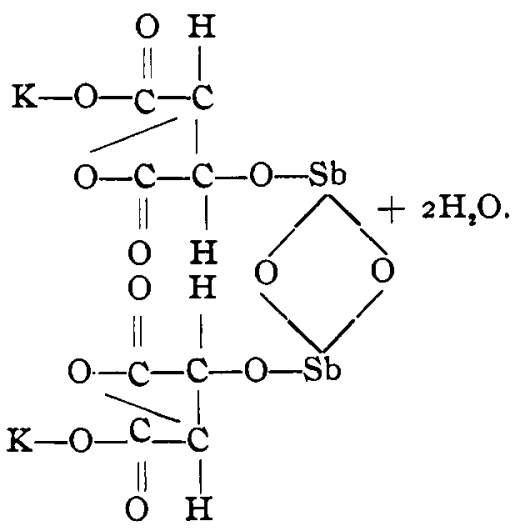

(Molecular weight $[305.15]_{2}$.) (Tartar emetic, lactone.)

\section{SUMMARY.}

It has been shown that the discrepancy between tartar emetic and arsenious oxide solutions lies in the ready tendency of the tartar emetic to lose its water of crystallization. If in fine crystalline or powdery form, tartar emetic loses its water of crystallization slowly in the air, more rapidly over sulphuric acid in an ordinary or vacuum desiccator, and somewhat upon standing in closed bottles; at a temperature of $128^{\circ}-130^{\circ}$, it begins to form an anhydride losing one-half molecule of water; at $160^{\circ}$ I $65^{\circ}$ it loses another molecule of water and becomes doubly anhydride. The formation of these anhydrides seems best explained structurally by attaching the antimony to the hydroxyl rather than to the carboxyl end of tartar emetic. If tartar emetic be recrystallized in medium-sized crystals, filtered, washed two or three times with distilled water, drained under suction for from five to ten minutes, and exposed at a temperature not exceeding $25^{\circ}$ for a period of from three to four hours to the action of a dry atmosphere, decinormal solutions of the salt thus freshly prepared will correspond exactly to decinormal solutions of arsenious oxide. 